•综述・

\title{
外来入侵植物的根系受养行为研究进展
}

\author{
韩 雪 苏锦权 姚娜娜 陈宝明* \\ (中山大学生命科学学院/有害生物控制与资源利用国家重点实验室, 广州 510275)
}

\begin{abstract}
摘要: 土壤养分分布具有高度空间异质性, 植物的根系受养行为是其对土壤养分异质性的一种适应。不同植物为 了适应养分异质性会产生不同的根系受养行为, 通过调整自身的根系受养范围、受养精度和受养速度来更好地吸 收利用土壤中的养分。外来植物与本地植物的竞争是决定其成功入侵的重要因素, 土壤养分等环境因素会影响它 们之间的竞争关系。近年来, 外来入侵植物的受养行为逐渐受到人们的关注, 关于入侵植物根系受养行为的研究 成果陆续出现: (1)总体来看, 外来入侵植物具有较强的根系受养能力, 但根系受养范围与受养精度之间的权衡关 系还不确定; (2)营养异质性会影响入侵植物与本地植物之间的竞争, 反过来, 二者之间的竞争也会影响根系受养 行为对营养异质性的响应; (3)从枝菌根真菌(arbuscular mycorrhizal fungi, AMF)能够提高入侵植物的根系受养能 力, 外来植物入侵能够改变入侵植物对AMF的偏好性, 形成AMF对入侵的正反馈作用, 而本地植物与AMF的相互 作用也会影响入侵植物的竞争力。未来还应加强营养异质环境下种间竞争和AMF共生对入侵植物根系受养行为的 影响机制研究, 以及全球变化背景下入侵植物根系受养行为的变化与机制方面的研究, 可以更深入地认识外来植 物的受养行为在其成功入侵中的作用, 并为利用营养调控来防控入侵植物提供理论依据。
\end{abstract}

关键词：根系受养; 外来入侵植物; 营养异质性; 丛枝菌根真菌; 竞争; 根系分布

\section{Advances in root foraging behavior of exotic invasive plants}

\author{
Xue Han, Jinquan Su, Nana Yao, Baoming Chen ${ }^{*}$ \\ School of Life Sciences, State Key Laboratory of Biocontrol, Sun Yat-Sen University, Guangzhou 510275
}

\begin{abstract}
Nutrient distribution within soils is highly spatially heterogenous, forcing plant root foraging behavior to adapt to heterogeneous soil nutrients. By adjusting root foraging scales, foraging precision and rate, plants can maximize soil nutrients. Successful invaders are heavily influenced by soil nutrients and other environmental conditions (e.g. water content, light, etc.) as successful invasion depends on the successful competitive interaction of an exotic species with a native species. In recent years, the foraging behavior of exotic invasive plants has attracted more attention, and studies are increasing worldwide. To date, foraging behavior studies have found: (1) Invasive plants have increased root foraging abilities compared to native plants, although the trade-off between foraging scale and precision are uncertain. (2) Soil nutrient heterogeneity influences the competitive outcome between invasive and native plants, as competition alters root foraging strategy. (3) Arbuscular mycorrhizal fungi (AMF) facilitate root foraging of invasive plants, and invasive plants experience positive feedbacks with invasion by altering their AMF preference. Additionally, the interaction between AMF and native plants can influence the competitive ability of invasive plants. Further studies are necessary to understand the relationship between interspecific competition and AMF on root foraging behavior of invasive plants in heterogeneous nutrient conditions. Due to global change, root foraging strategies and related mechanisms of invasive species may be altering. Increased research on root foraging behavior in successful exotic plant invasions can provide a greater understanding of these mechanisms and a theoretical basis for control and management of invasive plants through nutrient regulation.
\end{abstract}

Key words: root foraging; exotic invasive plants; soil nutrient heterogeneity; arbuscular mycorrhizal fungi; competition; root distribution 
土壤养分分布具有高度的空间异质性(Farley \& Fitter, 1999; Levine et al, 2016), 植物为了适应土 壤养分异质性，一般会通过根系形态和生理上的一 系列可塑性变化来探寻、获取、利用养分, 即植物 根系的受养行为(foraging behavior) (Hutchings \& de Kroon, 1994)。植物根系的受养行为主要包括根系的 受养范围(foraging scale)、受养精度(foraging precision)与受养速度(foraging rate) (Rajaniemi \& Reynolds, 2004; de Kroon \& Mommer, 2006)。受养范围 是指植物根系在富营养斑块中延伸的广度, 通常用 富营养斑块内根系的生物量或根系密度(单位体积 土壤中根的长度)表示; 受养精度指植物根系对不 同斑块的选择性, 通常用富营养斑块和贫营养斑块 中根系生物量或根系密度的比值来表示; 受养速度 指根系到达富营养斑块的时间 (Rajaniemi \& Reynolds, 2004)。

植物根系的受养行为主要是由激素调控。例如 在低磷(P)环境下, 拟南芥(Arabidopsis thaliana)通 过诱导生长素合成关键酶的表达来增加生长素的 合成, 进而调控根毛伸长及次生根的生长实现植物 根系受养(Bhosale et al, 2018); 在缺氮(N)条件下, 添加硝酸盐能瞬时上调植物体内生长素受体信号 的表达, 增加初生根尖端的生长素累积和敏感性, 促进植物根生长和养分吸收(Giehl \& von Wiren, 2014)。这些研究表明, 植物在营养缺乏的环境中主 要通过调节生长素的合成来调控根系受养行为, 进 而达到吸收养分的目的。

外来生物入侵被认为是仅次于生境破坏导致 全球生物多样性下降的第二大因素, 严重威胁着生 态系统健康(Ehrenfeld, 2010; Vilà et al, 2011)。外来 植物之所以能够成功入侵, 一方面与其生长速度 快、繁殖能力强、竞争力强、化感作用强以及高效 的资源捕获与利用能力 (Callaway \& Aschehoug, 2000; Funk \& Vitousek, 2007; van Kleunen et al, 2010)等特性有关, 另一方面与入侵地的养分、温 度、光照等环境因素有关(Bradley et al, 2010; Zhang et al, 2017b; Broadbent et al, 2018)。入侵物种到达新 生境后, 一般先占领条件较为适宜的环境, 成功建 群后再逐渐往外扩散。

高的表型可塑性是许多外来植物成功入侵的 原因之一(Richards et al, 2016)。面对土壤营养异质 性环境, 外来入侵植物通常能够更快地将根生物量
分布在富营养斑块，以提高其营养吸收能力，优先 获取资源并降低其他物种的可利用资源，从而获得 生长优势并成功入侵(Drenovsky et al, 2008; James et al, 2009)。近年来, 人们越来越关注外来入侵植物 的根系受养行为(Keser et al, 2014; Chen et al, 2018), 本文主要从外来入侵植物的根系受养特性及权衡 关系(trade-off)、入侵植物的直接根系受养行为与竞 争、广义的根系受养行为(丛枝菌根真菌(arbuscular mycorrhizal fungi, AMF)与植物的养分吸收) 3 个方 面来综述相关研究进展, 并讨论未来的研究方向。

\section{外来入侵植物的根系受养特性及权衡关示}

土壤养分异质性给外来入侵植物提供了更多 利用资源的机会，有利于其入侵(Davis \& Pelsor, 2001; Melbourne et al, 2007)。Drenovsky等(2008)比 较了 4 种本地植物和 4 种外来入侵植物的根系受养 能力, 结果发现入侵植物的地上、地下生物量是本 地植物的2-3倍，且在营养异质性处理中入侵植物 的根系密度高达 $60 \mathrm{~km} / \mathrm{m}^{3}$, 显著高于本地植物(约 $30 \mathrm{~km} / \mathrm{m}^{3}$ ); 而在均质营养条件下, 入侵植物的根系 密度为 $30 \mathrm{~km} / \mathrm{m}^{3}$ 左右，不考虑根生物量很小的 2 种 本地植物, 其余 2 种本地植物的根系密度也只有 15 $\mathrm{km} / \mathrm{m}^{3}$ 左右，远低于入侵植物。James等(2009)研究 了3 种入侵非草本植物、3 种本地丛生禾草与 3 种本 地非草本植物的根系受养行为, 结果显示入侵植物 的根系密度大于本地的草本和非草本植物, 即入侵 植物根系受养范围较大。无论是在营养异质性土壤 还是在营养均质性土壤中, 入侵植物的氮吸收速率 都高于本地植物。他们认为, 入侵植物的氮吸收速 率、相对生长速率和根生物量之间存在正相关关系, 但与根系受养精度不存在明显的正相关关系(James et al, 2009)。这在一定程度上说明在营养异质性的 土壤中, 入侵植物的氮吸收速率与根生物量能够同 步增加, 进而促进植物根系受养范围的增加。

Keser等(2014)利用线性混合模型分析了菊科、 伞形科、禾本科、玄参科、唇形科和石竹科的 6 对 入侵植物和外来非入侵植物对异质性土壤的不同 反应。研究发现, 异质性土壤中入侵植物根系总生 物量要高于外来非入侵植物, 且入侵植物在营养异 质性条件下富营养斑块中的根生物量显著高于贫 营养斑块，而外来非入侵植物的根生物量分配则没 有显著变化。近期研究发现，外来入侵植物薇甘菊 
(Mikania micrantha)和白花鬼针草(Bidens pilosa var. radiata)表现出较大的受养范围和较低的受养精度, 而与之伴生的本地植物鸡屎藤(Paederia scandens)、 夜香牛(Vernonia cinerea)则表现出较高的受养精度 和较小的受养范围(Chen et al, 2018)。然而, Keser等 (2015)分析了 24 种入侵植物和外来非入侵植物的根 系受养特征, 发现入侵植物的受养范围与外来非入 侵植物没有显著差别, 而用根直径作为根系受养特 性的分析指标时, 入侵植物比非入侵植物具有更强 的根系受养能力, 这说明采用不同的指标对根系受 养特性进行分析计算有可能会得到不同的结论。

一般来说，植物根系受养范围和受养精度之间 存在权衡关系, 植物会根据自身与环境的情况来选 择更适宜植物生长的根系受养策略(Campbell et al, 1991)。Kembel等(2008)用整合分析(meta-analysis) 的方法研究发现, 当物种数量比较少时, 受养范围 和受养精度之间呈现明显的负相关关系(即存在权 衡关系), 而当物种数量较多时, 二者之间的相关关 系消失, 说明物种数量的多少会影响受养范围和精 度之间的权衡关系。然而, Grime (2007)认为植物根 系的受养范围与受养精度之间并不存在权衡关系。 近期研究发现, 在营养异质性环境下, 入侵植物与 本地植物之间存在 “受养范围一受养精度”的权衡关 系: 入侵植物薇甘菊和白花鬼针草的根系受养范围 较大, 精度较小, 本地植物夜香牛与鸡屎藤则刚好 相反(Chen et al, 2018)。

总体来看, 有些入侵植物是通过较大的受养范 围来提高养分的吸收能力从而达到快速生长, 有些 通过提高受养精度来增强与本地植物竞争养分的 能力, 有些入侵植物的受养范围和受养精度都优于 本地植物。植物根系的受养范围与受养精度之间是 否存在权衡关系目前还不清楚, 入侵植物与本地植 物的受养范围与受养精度的权衡关系是否有差异 值得关注, 需要进一步的研究。

\section{2 入侵植物的根系受养行为及其竞争能力}

入侵植物与本地植物之间的竞争关系是决定 外来植物能否成功入侵的关键(Levine et al, 2004; 万方浩等, 2011; Golivets \& Wallin, 2018)。外来入侵 植物直接与本地植物竞争养分、水分、光照等资源 (Lopez-Zamora et al, 2004; Gioria \& Osborne, 2014; Hulvey \& Teller, 2018), 而养分、光照、温度等环境
因素也会影响入侵植物和本地植物之间的竞争 (Suding et al, 2004; Bradley et al, 2010; Verlinden et al, 2014; Broadbent et al, 2018)。

土壤营养异质性会影响植物间的竞争, 同时植 物也能根据土壤营养分布状况与邻体植物的信息 来调整自身的根系分布(Cahill et al, 2010; Mommer et al, 2012)。Zhou等(2012) 通过对空心莲子草 (Alternanthera philoxeroides)的研究发现, 土壤营养 异质性并没有改变其种内竞争的强度, 这可能是因 为同种植物相互识别并且避免种内竞争, 在进化上 更为有利。单独种植的苟麻(Abutilon theophrasti)根 系分布对土壤营养的分布没有响应，但是与同种植 物共植且两植株之间存在富营养斑块时，它们的根 系会倾向于重叠，进而加剧其种内竞争(Cahill et al, 2010)。黑麦草(Lolium perenne)与草地早熟禾(Poa pratensis)的根系受养行为只有在两者竞争时才显 现出来, 单独种植时受养行为并不明显(Hodge et al, 1999; Hodge, 2004, 2006)。

在营养异质性的土壤中, 两种植物竞争能力的 相对大小通常会影响其根系受养行为: 竞争能力较 强的植物其受养行为并不受土壤营养异质性的影 响, 但竞争能力较弱的植物在存在竞争时, 会减少 富营养斑块中的根系分布，而增加贫营养斑块中的 根系生物量及营养吸收速率, 以补偿富营养斑块中 的高竞争压力(Mommer et al, 2012)。

可见，营养异质性会影响植物之间的竞争关系， 反过来邻体植物的竞争也会影响入侵植物对营养 异质性的响应，进而影响其根系的受养行为。营养 异质性条件下植物根系受养行为与植物竞争之间 的相互作用更值得关注。

\section{3 广义的根系受养行为- AMF与植物白 吸收}

\subsection{AMF促进植物的养分吸收能力}

大多数陆地植物(尤其是草本植物)能与 $\mathrm{AMF}$ 共 生(Smith \& Read, 2008; 何新华等, 2012; van der Heijden et al, 2015), AMF不仅可以帮助植物捕获磷 (van der Heijden et al, 2003; Hodge, 2004; Cheng et $\mathrm{al}, 2019)$, 还可以帮助植物捕获氮(Leigh et al, 2009) 和钾 $(K)$ 等养分(Estrada et al, 2013)。研究发现，接种 AMF后植物体内的钾含量显著高于未接种 AMF的 植物(Estrada et al, 2013), AMF处理的植株体内的磷 
和氮含量分别是未受AMF处理的25倍和10倍(van der Heijden et al, 2003)。尿素循环代谢臂(catabolic arm of the urea cycle)可以使真菌在碳损失最小的条 件下将氮转移到宿主植物, 说明植物通过AMF共生 能够有效地将土壤中大量的氮转移到根系 (Govindarajulu et al, 2005)。AMF还可以从有机物质 中捕获氮并将其转移到植物体中 (Leigh et al, 2009)。

\subsection{AMF 在入侵植物根系受养中的作用}

外来入侵植物与土著 AMF共生, 有助于其对 氮和磷的吸收, 从而有利于其入侵(Reinhart \& Callaway, 2006; Harner et al, 2010; 于文清等, 2012; Zhang et al, 2017a)。Bunn等(2015)用整合分析的方 法研究了 67 篇论文中 70 种本地植物和 55 种入侵植 物与 AMF共生的情况, 结果表明入侵植物与本地植 物的AMF丰度没有显著差异, 但在78\%的研究中与 入侵植物共生的 $\mathrm{AMF}$ 群落和与本地植物共生的 AMF群落明显不同, 并且入侵植物会降低本地植物 的AMF侵染率, 说明入侵植物与本地植物的竞争明 显改变了它们与AMF的相互作用。然而, 在美国半 湿润草地的研究发现, 菌根侵染率与入侵植物地上 生物量没有显著的正相关关系, 而与本地植物呈显 著正相关, 说明植物的菌根依赖性 (mycorrhizal dependence) 可能在入侵植物与本地植物的竞争中 起重要作用(Sielaff et al, 2019)。Elizabeth等(2009) 发现入侵北美的贯叶连尧(Hypericum perforatum)种 群比在原产地欧洲的种群AMF侵染率低, 说明贯叶 连尧通过降低根部对 AMF的依赖性达到入侵繁殖 的目的。Zhang 等 (2010) 发现加拿大一枝黄花 (Solidago canadensis) 的入侵促进了 AMF中地球囊 霉(Glomus geosporum)的生长, 而抑制了摩西球囊 霉(G. mosseae)的生长, 入侵引起的地球囊霉的增 加可以促进外来植物的生长, 而摩西球囊霉的减少 则不利于本地植物的生长, 最终加拿大一枝黄花通 过AMF对其入侵产生正反馈作用, 说明有些入侵植 物能够改变自身与本地植物对AMF的偏好性, 形成 入侵的正反馈。

\subsection{AMF的碳转移作用及AMF对本地植物养分竞 争的影响}

由于 $A M F$ 为植物提供养分的前提是需要宿主 植物提供更多的碳源为代价, 即宿主通过AMF共生 间接受养需要更高的碳成本, 因此宿主植物要权衡
是否付出更多的碳成本而通过AMF共生体来获取 养分(Treseder et al, 2018)。然而, 研究发现AMF还 会影响植物个体之间的碳转移, AMF不仅能够通过 共生体促进入侵植物斑点矢车菊 (Centaurea maculosa) 的生长, 而且能够间接地将邻近的本地植 物伊达霍恩斯羊茅(Festuca idahoensis)的C转移到 入侵植物斑点矢车菊(Carey et al, 2004)。

本地植物与土著 AMF之间的共生互利会帮助 本地植物吸收养分, 提高本地植物对外来入侵植物 的竞争力和抵抗力(Kisa et al, 2007; Dickie et al, 2017)。近期研究表明AMF可以促进本地植物金盏 银盘(Bidens biternata)对磷的吸收进而提高其与外 来入侵物植物白花鬼针草的竞争力(Cheng et al, 2019)。可见，本地植物与AMF的相互作用不容忽视, 其相互作用会影响本地植物对入侵植物的抵抗力。

\section{4 土壤养分对植物与 $\mathrm{AMF}$ 共生关系的影响}

植物通过AMF共生影响其对养分的捕获吸收, 反过来土壤养分可以直接或间接影响植物的 $\mathrm{AMF}$ 侵染、菌根共生体系发育、产狍和分布，土壤养分 还会改变 AMF与宿主植物之间的关系(Johnson, 2010; Dickie et al, 2017; 向丹等, 2017; Treseder et al, 2018)。在养分限制的条件下, 菌根真菌为宿主提供 养分的有益作用大于宿主为菌根真菌提供碳源的 成本, 菌根真菌与宿主植物形成互利共生关系 (Bever et al, 2010; Johnson et al, 2010)。在养分充足 的条件下，菌根真菌为宿主提供养分的有益作用小 于宿主为菌根真菌提供碳源的成本, 菌根真菌与宿 主植物形成寄生关系(Schulz \& Boyle, 2005)。添加 养分会降低植物AMF的侵染, 养分充足时植物更倾 向于通过根系而不是通过 $\mathrm{AMF}$ 吸收养分(Eissenstat et al, 2015)。

\section{研究展望}

入侵植物的根系受养行为是非常复杂的生理 过程, 不同植物会产生不同的根系受养行为适应营 养异质性, 入侵植物与本地植物的竞争会影响植物 根系的受养行为, 其中还包含着自身以及与各种环 境因素之间的权衡。目前, 关于外来入侵植物根系 受养行为的研究还处于起步发展阶段, 还有许多需 要研究解答的问题。

首先，营养异质环境下本地植物的竞争对入侵 植物根系的受养能力有何影响? 入侵植物根系直 
接受养与植物-AMF共生吸收养分之间有何关联, 二者对外来入侵植物的竞争力有何影响, 作用机制 是什么?

其次, 全球气候变化 $\left(\right.$ 气温升高、大气 $\mathrm{CO}_{2}$ 浓度 升高、氮沉降、极端气候变化事件等)及其他环境因 素(如光照、土壤水分等)会改变植物－微生物之间的 相互作用, 未来应加强全球变化背景下入侵植物根 系受养行为的变化与机制方面的研究, 进一步探究 根系受养行为与AMF等土壤微生物的相互作用。

外来入侵植物的根系受养行为是一个复杂的 过程, 相关研究的数量和深度还远远不够, 需要我 们更多关注和探讨, 更好地阐明外来植物的受养行 为、入侵力、竞争力对环境变化的响应及其机制, 为 外来入侵植物的管控和入侵地的恢复提供依据。

\section{参考文献}

Bever JD, Dickie IA, Facelli E, Facelli JM, Klironomos J, Moora M, Rillig MC, Stock WD, Tibbett M, Zobel M (2010) Rooting theories of plant community ecology in microbial interactions. Trends in Ecology \& Evolution, 25, 468-478.

Bhosale R, Giri J, Pandey BK, Giehl RFH, Hartmann A, Traini R, Truskina J, Leftley N, Hanlon M, Swarup K, Rashed A, Voss U, Alonso J, Stepanova A, Yun J, Ljung K, Brown KM, Lynch JP, Dolan L, Vernoux T, Bishopp A, Wells D, von Wiren N, Bennett MJ, Swarup R (2018) A mechanistic framework for auxin dependent Arabidopsis root hair elongation to low external phosphate. Nature Communications, 9, 1409.

Bradley BA, Blumenthal DM, Wilcove DS, Ziska LH (2010) Predicting plant invasions in an era of global change. Trends in Ecology \& Evolution, 25, 310-318.

Broadbent AAD, Stevens CJ, Ostle NJ, Orwin KH (2018) Biogeographic differences in soil biota promote invasive grass response to nutrient addition relative to co-occurring species despite lack of belowground enemy release. Oecologia, 186, 611-620.

Bunn RA, Ramsey PW, Lekberg Y (2015) Do native and invasive plants differ in their interactions with arbuscular mycorrhizal fungi? A meta-analysis. Journal of Ecology, 103, 1547-1556.

Cahill JF, McNickle GG, Haag JJ, Lamb EG, Nyanumba SM, Clair CCS (2010) Plants integrate information about nutrients and neighbors. Science, 328, 1657.

Callaway RM, Aschehoug ET (2000) Invasive plants versus their new and old neighbors: A mechanism for exotic invasion. Science, 290, 521-523.

Campbell BD, Grime JP, Mackey JML (1991) A trade-off between scale and precision in resource foraging. Oecologia,
87, 532-538.

Carey EV, Marler MJ, Callaway RM (2004) Mycorrhizae transfer carbon from a native grass to an invasive weed: Evidence from stable isotopes and physiology. Plant Ecology, 172, 133-141.

Chen BM, Su JQ, Liao HX, Peng SL (2018) A greater foraging scale, not a higher foraging precision, may facilitate invasion by exotic plants in nutrient-heterogeneous conditions. Annals of Botany, 121, 561-569.

Cheng JK, Yue MF, Yang HR, Chen BM, Xin GR (2019) Do arbuscular mycorrhizal fungi help the native species Bidens biternata resist the invasion of Bidens alba? Plant and Soil, 444, 443-455.

Davis MA, Pelsor M (2001) Experimental support for a resource-based mechanistic model of invasibility. Ecology Letters, 4, 421-428.

de Kroon H, Mommer L (2006) Root foraging theory put to the test. Trends in Ecology \& Evolution, 21, 113-116.

Dickie IA, Bufford JL, Cobb RC, Desprez-Loustau ML, Grelet G, Hulme PE, Klironomos J, Makiola A, Nunez MA, Pringle A, Thrall PH, Tourtellot SG, Waller L, Williams NM (2017) The emerging science of linked plant-fungal invasions. New Phytologist, 215, 1314-1332.

Drenovsky RE, Martin CE, Falasco MR, James JJ (2008) Variation in resource acquisition and utilization traits between native and invasive perennial forbs. American Journal of Botany, 95, 681-687.

Ehrenfeld JG (2010) Ecosystem consequences of biological invasions. Annual Review of Ecology, Evolution, and Systematics, 41, 59-80.

Eissenstat DM, Kucharski JM, Zadworny M, Adams TS, Koide RT (2015) Linking root traits to nutrient foraging in arbuscular mycorrhizal trees in a temperate forest. New Phytologist, 208, 114-124.

Elizabeth KS, James DB, John LM (2009) Evidence for the evolution of reduced mycorrhizal dependence during plant invasion. Ecology, 90, 1055-1062.

Estrada B, Aroca R, Maathuis FJ, Barea JM, Ruiz-Lozano JM (2013) Arbuscular mycorrhizal fungi native from a Mediterranean saline area enhance maize tolerance to salinity through improved ion homeostasis. Plant, Cell \& Environment, 36, 1771-1782.

Farley RA, Fitter AH (1999) Temporal and spatial variation in soil resources in a deciduous woodland. Journal of Ecology, 87, 688-696.

Funk JL, Vitousek PM (2007) Resource-use efficiency and plant invasion in low-resource systems. Nature, 446, 1079-1081.

Giehl RF, von Wiren N (2014) Root nutrient foraging. Plant Physiology, 166, 509-517.

Gioria M, Osborne BA (2014) Resource competition in plant invasions: Emerging patterns and research needs. Frontiers in Plant Science, 5, 501. 
Golivets M, Wallin KF (2018) Neighbour tolerance, not suppression, provides competitive advantage to non-native plants. Ecology Letters, 21, 745-759.

Govindarajulu M, Pfeffer PE, Jin H, Abubaker J, Douds DD, Allen JW, Bücking H, Lammers PJ, Shachar-Hill Y (2005) Nitrogen transfer in the arbuscular mycorrhizal symbiosis. Nature, 435, 819-823.

Grime JP (2007) The scale-precision trade-off in spacial resource foraging by plants: Restoring perspective. Annals of Botany, 99, 1017-1021.

Harner MJ, Mummey DL, Stanford JA, Rillig MC (2010) Arbuscular mycorrhizal fungi enhance spotted knapweed growth across a riparian chronosequence. Biological Invasions, 12, 1481-1490.

He XH, Duan YH, Chen YL, Xu MG (2012) Study of mycorrhiza in China for 60 years: Past, present and future. Scientia Sinica Vitae, 42, 431-454. (in Chinese with English abstract) [何新华, 段英华, 陈应龙, 徐明岗 (2012) 中国 菌根研究60年: 过去、现在和将来. 中国科学: 生命科学, 42, 431-454.]

Hodge A (2004) The plastic plant: Root responses to heterogeneous supplies of nutrients. New Phytologist, 162, 9-24.

Hodge A (2006) Plastic plants and patchy soils. Journal of Experimental Botany, 57, 401-411.

Hodge A, Robinson D, Griffiths BS, Fitter AH (1999) Why plants bother: Root proliferation results in increased nitrogen capture from an organic patch when two grasses compete. Plant, Cell \& Environment, 22, 811-820.

Hulvey KB, Teller BJ (2018) Site conditions determine a key native plant's contribution to invasion resistance in grasslands. Ecology, 99, 1257-1264.

Hutchings MJ, de Kroon H (1994) Foraging in plants: The role of morphological plasticity in resource acquisition. Advances in Ecological Research, 25, 159-238.

James J, Mangold J, Sheley R, Svejcar T (2009) Root plasticity of native and invasive Great Basin species in response to soil nitrogen heterogeneity. Plant Ecology, 202, 211-220.

Johnson NC (2010) Resource stoichiometry elucidates the structure and function of arbuscular mycorrhizas across scales. New Phytologist, 185, 631-647.

Kembel SW, de Kroon H, Cahill JF Jr, Mommer L (2008) Improving the scale and precision of hypotheses to explain root foraging ability. Annals of Botany, 101, 1295-1301.

Keser LH, Dawson W, Song YB, Yu FH, Fischer M, Dong M, van Kleunen M (2014) Invasive clonal plant species have a greater root-foraging plasticity than non-invasive ones. Oecologia, 174, 1055-1064.

Keser LH, Visser EJ, Dawson W, Song YB, Yu FH, Fischer M, Dong M, van Kleunen M (2015) Herbaceous plant species invading natural areas tend to have stronger adaptive root foraging than other naturalized species. Frontiers in Plant Science, 6, 237.

Kisa M, Sanon A, Thioulouse J, Assigbetse K, Sylla S,
Spichiger R, Dieng L, Berthelin J, Prin Y, Galiana A, Lepage M, Duponnois R (2007) Arbuscular mycorrhizal symbiosis can counterbalance the negative influence of the exotic tree species Eucalyptus camaldulensis on the structure and functioning of soil microbial communities in a Sahelian soil. FEMS Microbiology Ecology, 62, 32-44.

Leigh J, Hodge A, Fitter AH (2009) Arbuscular mycorrhizal fungi can transfer substantial amounts of nitrogen to their host plant from organic material. New Phytologist, 181, 199-207.

Levine JM, Adler PB, Yelenik SG (2004) A meta-analysis of biotic resistance to exotic plant invasions. Ecology Letters, 7, 975-989.

Levine NM, Zhang K, Longo M, Baccini A, Phillips OL, Lewis SL, Alvarez-Dávila E, Segalin de Andrade AC, Brienen RJW, Erwin TL, Feldpausch TR, Monteagudo Mendoza AL, Nuñez Vargas P, Prieto A, Silva-Espejo JE, Malhi Y, Moorcroft PR (2016) Ecosystem heterogeneity determines the ecological resilience of the Amazon to climate change. Proceedings of the National Academy of Sciences, USA, 113, 793-797.

Lopez-Zamora I, Comerford NB, Muchovej RM (2004) Root development and competitive ability of the invasive species Melaleuca quinquenervia (Cav.) ST Blake in the South Florida flatwoods. Plant and Soil, 263, 239-247.

Melbourne BA, Cornell HV, Davies KF, Dugaw CJ, Elmendorf S, Freestone AL, Hall RJ, Harrison S, Hastings A, Holland M, Holyoak M, Lambrinos J, Moore K, Yokomizo H (2007) Invasion in a heterogeneous world: Resistance, coexistence or hostile takeover? Ecology Letters, 10, 77-94.

Mommer L, van Ruijven J, Jansen C, van de Steeg HM, de Kroon H (2012) Interactive effects of nutrient heterogeneity and competition: Implications for root foraging theory? Functional Ecology, 26, 66-73.

Rajaniemi TK, Reynolds HL (2004) Root foraging for patchy resources in eight herbaceous plant species. Oecologia, 141, 519-525.

Richards CL, Bossdorf O, Muth NZ, Gurevitch J, Pigliucci M (2006) Jack of all trades, master of some? On the role of phenotypic plasticity in plant invasions. Ecology Letters, 9, 981-993.

Reinhart KO, Callaway RM (2006) Soil biota and invasive plants. New Phytologist, 170, 445-457.

Schulz B, Boyle C (2005) The endophytic continuum. Mycological Research, 109, 661-686.

Sielaff AC, Polley HW, Fuentes-Ramirez A, Hofmockel K, Wilsey BJ (2019) Mycorrhizal colonization and its relationship with plant performance differs between exotic and native grassland plant species. Biological Invasions, 21, 1981-1991.

Smith SE, Read DJ (2008) Mycorrhizal Simbiosis, 3rd edn. Academic Press, London.

Suding KN, LeJeune KD, Seastedt TR (2004) Competitive 
impacts and responses of an invasive weed: Dependencies on nitrogen and phosphorus availability. Oecologia, 141, 526-535.

Treseder KK, Allen EB, Egerton-Warburton LM, Hart MM, Klironomos JN, Maherali H, Tedersoo L (2018) Arbuscular mycorrhizal fungi as mediators of ecosystem responses to nitrogen deposition: A trait-based predictive framework. Journal of Ecology, 106, 480-489.

van der Heijden MGA, Martin FM, Selosse MA, Sanders IR (2015) Mycorrhizal ecology and evolution: The past, the present, and the future. New Phytologist, 205, 1406-1423.

van der Heijden MGA, Wiemken A, Sanders IR (2003) Different arbuscular mycorrhizal fungi alter coexistence and resource distribution between co-occurring plant. New Phytologist, 157, 569-578.

van Kleunen M, Weber E, Fischer M (2010) A meta-analysis of trait differences between invasive and non-invasive plant species. Ecology Letters, 13, 235-245.

Verlinden M, de Boeck HJ, Nijs I (2014) Climate warming alters competition between two highly invasive alien plant species and dominant native competitors. Weed Research, 54, 234-244.

Vilà M, Espinar JL, Hejda M, Hulme PE, Jarošík V, Maron JL, Pergl J, Schaffner U, Sun Y, Pyšek P (2011) Ecological impacts of invasive alien plants: A meta-analysis of their effects on species, communities and ecosystems. Ecology Letters, 14, 702-708.

Wan FH, Xie BY, Yang GQ (2011) Invasion Biology. Science Press, Beijing. (in Chinese) [万方浩, 谢丙炎, 杨国庆 (2011) 入侵生物学. 科学出版社, 北京.]

Xiang D, Xu TL, Li H, Chen BD (2017) Ecological distribution of arbuscular mycorrhizal fungi and the influencing factors.
Acta Ecologica Sinca, 37, 3597-3606. (in Chinese with English abstract) [向丹, 徐天乐, 李欢, 陈保冬 (2017) 丛 枝菌根真菌的生态分布及其影响因子研究进展. 生态学 报, 37, 3597-3606.]

Yu WQ, Liu WX, Gui FR, Liu WZ, Wan FH, Zhang LL (2012) Invasion of exotic Ageratina adenophora Sprengel alters soil physical and chemical characteristics and arbuscular mycorrhizal fungus community. Acta Ecologica Sinica, 32, 7027-7035. (in Chinese with English abstract) [于文清, 刘 万学, 桂福荣, 刘文志, 万方浩, 张利莉 (2012) 外来植 物紫茎泽兰入侵对土壤理化性质及丛枝菌根真菌(AMF) 群落的影响. 生态学报, 32, 7027-7035.]

Zhang FJ, Li Q, Chen FX, Xu HY, Inderjit, Wan FH (2017a) Arbuscular mycorrhizal fungi facilitate growth and competitive ability of an exotic species Flaveria bidentis. Soil Biology and Biochemistry, 115, 275-284.

Zhang H, Chang R, Guo X, Liang X, Wang R, Liu J (2017b) Shifts in growth and competitive dominance of the invasive plant Alternanthera philoxeroides under different nitrogen and phosphorus supply. Environmental and Experimental Botany, 135, 118-125.

Zhang Q, Yang R, Tang J, Yang H, Hu S, Chen X (2010) Positive feedback between mycorrhizal fungi and plants influences plant invasion success and resistance to invasion. PLoS ONE, 5, e12380.

Zhou J, Dong BC, Alpert P, Li HL, Zhang MX, Lei GC (2012) Effects of soil nutrient heterogeneity on intraspecific competition in the invasive, clonal plant Alternanthera philoxeroides. Annals of Botany, 109, 813-818.

(责任编委: 冯玉龙 责任编辑: 黄祥忠) 